\title{
Suspected Calcinosis Cutis and Demodicosis, Secondary to an Exogenous Steroid Administration in a Dog with Hypoadrenocorticism
}

\section{Davide Immediato*}

*Correspondence: Davide Immediato

Address: Rotating intern at Southfield Veterinary Specialists, Laindon, Essex, UK

e-mail $\bowtie$ : immedida@gmail.com

Received: 24 January 2020; Accepted: 03 February 2020

Copyright: (C) 2020 Immediato D. This is an open-access article distributed under the terms of the Creative Commons Attribution License, which permits unrestricted use, distribution, and reproduction in any medium, provided that the original work is properly cited.

\begin{abstract}
Hypoadrenocorticism is an uncommon, life-threatening, disease with immune-mediated aetiology, caused by reduced production of glucocorticoids and often also mineralocorticoids. The treatment is supplementation of cortisol, and aldosterone analogues if needed. High exogenous glucocorticoid supplementation can result in iatrogenic hyperadrenocorticism, development of calcinosis cutis or immune-suppression leading to demodicosis.

A 4-year-old female neutered Labrador retriever who was receiving treatment for hypoadrenocorticism, was presented for bilateral, symmetrical, well-demarcated areas of alopecia with multiple firm pale-yellow hard and gritty plaque-like lesions, erythematous papules and ulceration which had developed; six months later the dog was presented for generalised alopecia and erythema. Diagnostics investigation for the plaque lesions included cytological examination which revealed amorphous glassy and transparent material and evidence of crystals compatible with calcinosis cutis. Skin scrapes of the areas of alopecia and erythema revealed Demodex spp. A reduction of glucocorticoids resulted in resolution of calcinosis cutis and treatment with a fluralaner chewable tablet (Bravecto®, MSD) treated her demodicosis.
\end{abstract}

Keywords: Calcinosis Cutis, Demodicosis, Steroid, Hypoadrenocorticism

\section{Introduction}

Hypoadrenocorticism is an uncommon, life-threatening, disease (Van Lanen and Sande, 2014; Lathan and Thompson, 2018). It is commonly caused by immune-mediated destruction of the adrenal gland, with loss of the zona fasciculata and zona reticularis resulting in cortisol (glucocorticoid) deficiency, and loss of the zona glomerulosa resulting in aldosterone (mineralocorticoid) deficiency (Van Lanen and Sande, 2014; Lathan and Thompson, 2018). Therapy for typical hypoadrenocorticism consists of lifelong glucocorticoid and mineralocorticoid supplementation (Van Lanen and Sande, 2014; Lathan and Thompson, 2018). The physiological dose of glucocorticoids (prednisone or prednisolone) is 0.1-0.25 $\mathrm{mg} / \mathrm{kg} /$ day, although some dogs (particularly larger breeds) do well on $0.05 \mathrm{mg} / \mathrm{kg} /$ day (Peterson and Kintzer, 2009; Schaer, 2001; Van Lanen and Sande, 2014; Lathan and Thompson, 2018). Desoxycorticosterone pivalate (DOCP) is a long-lasting mineralocorticoid supplement, initially used at 
$2.2 \mathrm{mg} / \mathrm{kg}$ subcutaneously or intramuscular every 25 days (Van Lanen and Sande, 2014; Lathan and Thompson, 2018) and the dose can be adjusted based on electrolytes (Lathan and Thompson, 2018).

Excessive administration of corticosteroids can result in iatrogenic hyperadrenocorticism (Huang et al., 1999). This condition can be induced by topical, parenteral, or oral medications containing corticosteroid. The development of signs of glucocorticoid excess depends on the dosage and duration of the exposure (Huang et al., 1999) and can include hyperadrenocorticism and calcinosis cutis (CC) (Frazier et al., 1998; Huang et al., 1999, Hus et al., 2012, Jang et al., 2013) or parasite and bacterial infections (Coatesworth, 2011).

CC is an uncommon occurrence in which inorganic, insoluble mineral salts are deposited in the dermis, subcutis or, rarely, the epidermis (Frazier et al., 1998; Munday et al., 2005; Holahan et al., 2008; Hsu et al., 2012; Doerr et al., 2013; Jang et al., 2013; Tan et al., 2013; Volk et al., 2015). It is usually divided into four separate categories: metastatic, iatrogenic, dystrophic and idiopathic (Schaer et al., 2001; Munday et al., 2005; Hsu et al., 2012; Doerr et al., 2013; Jang et al., 2013; Tan et al., 2013; Volk et al., 2015). The most common affected sites are temporal area, dorsal aspect of the cervical region, dorsal midline, ventral aspect of the abdomen, and the groin and axillary regions (Schaer et al., 2001; Jang et al., 2013; Tan et al., 2013). Grossly, CC appears as pink to white, hard and gritty, well-demarcated skin lesions (Tan et al., 2013), consist of erythematous papules, plaques, or nodules that may ulcerate and become secondarily infected (Schaer et al., 2001; Tan et al., 2013; Jang et al., 2013). Treatment depends on the cause and treatment of the underlying disease (Schaer at al., 2001; Munday et al., 2005; Tan et al., 2013).

High levels of endogenous glucocorticoids which cause spontaneously occurring hyperadrenocorticism or inappropriate administration of exogenous glucocorticoids, can potentiate mite populations, increasing the numbers of Demodex canis resulting in demodicosis (Coatesworth, 2011) and, a diagnosis of adult-onset demodicosis, calls for investigations of a possible underlying cause (Coatesworth, 2011; Ferrer et al., 2014; Cereundolo, 2016; Koch, 2017).

\section{History}

A 4-year-old, female neutered Labrador retriever presented for further investigation of two skin conditions. She was up-to-date with routine vaccinations and was receiving monthly administration of imidacloprid/moxidectin spot-on (Advocate ${ }^{\circledR}$, Bayer) and every 3-monthly treatment with praziquantel tablets (Droncit $\AA$, Bayer).

In June 2017, the dog was diagnosed with typical hypoadrenocorticism and treatment with 2.2 
$\mathrm{mg} / \mathrm{Kg}$ of DOCP $25 \mathrm{mg} / \mathrm{ml}$ subcutaneous injection (Zycortal®, Dechra) and $0.5 \mathrm{mg} / \mathrm{kg} / \mathrm{day}$ of prednisolone $5 \mathrm{mg}$ tablet (Prednidale $®$, Dechra) was started. The dose was not changed for the following 6 months and the dog was clinically well. In January 2018, the dog was presented to one of my colleagues for investigation of an alopecic area on her flank with suppurative lesions; a seven-day treatment of amoxycillin/clavulanate, $250 \mathrm{mg}$ tablet $(10 \mathrm{mg} / \mathrm{Kg}) \mathrm{q} 12 \mathrm{~h}$ (Clavaseptin®, Vetoquinol) and with fusidic acid + betamethasone based topical cream q12h (Isaderm $\AA$, Dechra) was started and a presumptive diagnosis of seasonal flank alopecia was made. The dog was seen by another colleague the following month for the routine Zycortal $\AA$ injection. At this time the skin remained alopecic but there was no evidence of infection, the skin was less erythematous, and Clavaseptin ${ }^{\circledR} 250 \mathrm{mg}$ tablets, at $20 \mathrm{mg} / \mathrm{Kg}$ $\mathrm{q} 12 \mathrm{~h}$ was prescribed. The following month the dog presented to me for a routine Zycortal ${ }^{\circledR}$ injection, and, at that time, I was able to assess the skin condition and take over the case.

The dog developed a second skin condition about 6 months later from the first one, that will be discussed in the course of this case report.

\section{Clinical Examination}

At the time of the first skin condition the dog was bright and well in herself, her hypoadrenocorticism was well controlled, electrolytes values were within the desired reference interval, and no vomiting or diarrhoea was reported. Clinical examination was unremarkable except for the skin condition, body condition score 6/9 (WSAVA website) and body weight $24.7 \mathrm{Kg}$.

For the second skin condition the dog was fine in herself, clinical examination was unremarkable, except for the skin condition, body condition score 6/9 (WSAVA website) and body weight $25.3 \mathrm{Kg}$.

\section{Dermatological Examination}

At the time of the first presentation, the dog had bilaterally symmetrical well-demarcated areas of alopecia, which were roughly rectangular in shape of about $5 \times 15 \mathrm{~cm}$ on both sides of her rump. The skin was thick, with evidence of multiple, firm, pale-yellow, hard and gritty plaque-like lesions, with evidence of erythematous papules and ulceration. The dog was not pruritic according to the owner. Unfortunately, no pictures are available from the patient currently.

At the presentation for the second skin condition, there was alopecia and erythema interesting all four legs (from feet to elbow/stifle), face, ventral aspect of the neck and abdomen, there were also pustules, epidermal collarets, crusts and follicular casts (Figures 1, 2a, 2b, 3). The dog was pruritic. 


\section{Differential Diagnosis}

\section{For the first skin condition:}

- $\quad$ superficial/deep pyoderma

- dermatophytosis

- calcinosis cutis

For the second skin condition:

- demodicosis

- dermatophytosis

- $\quad$ superficial pyoderma

- cheyletiellosis

- $\quad$ sarcoptic mange

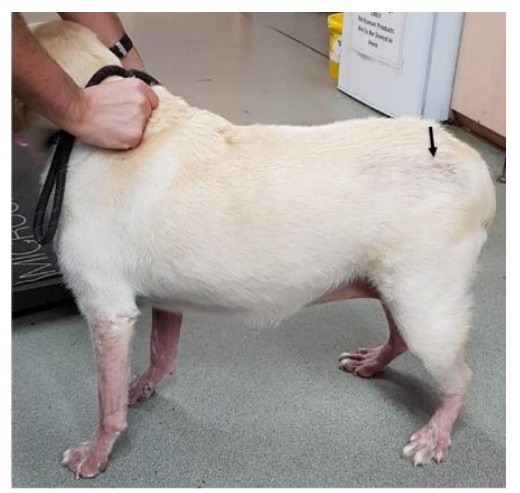

Figure 1: Presentation of the dog at the time of the second skin condition. Evidence of alopecia and erythema interesting the legs and abdomen. In this picture is also possible to observe partially the lesion, in regression, developed in course of suspected calcinosis cutis (black arrow).

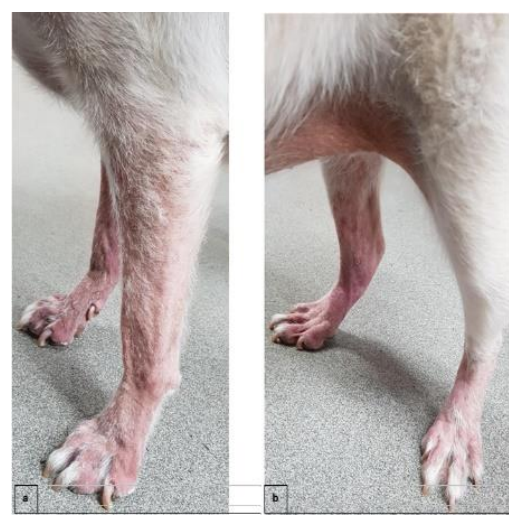

Figures 2: Particular of the front (a) and back (b) leg where is possible to observe the extension of the alopecia, the erythema and presence of scales. 


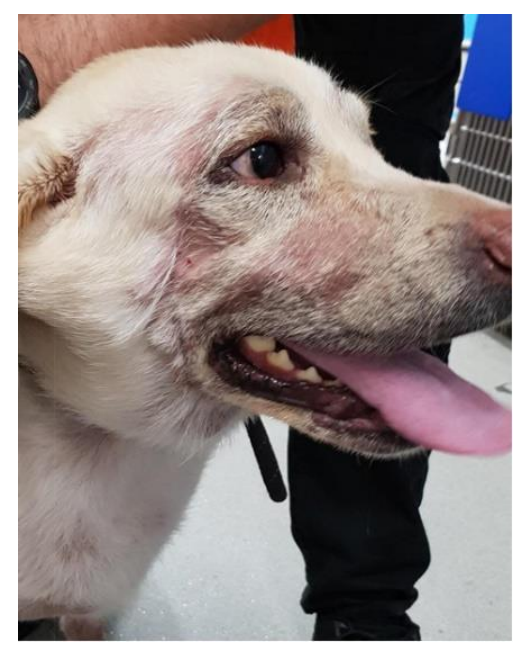

Figure 3: Particular of the face where is possible to observe the extension of the alopecia and erythema. Also, evidence of the lesion on the ventral part of the neck.

\section{Diagnostic Tests}

For the first skin condition, cytology, trichogram, wood lamp and dermatophyte PCR were performed.

- Trichogram: hairs were plucked from affected areas using forceps, placed on a slide with liquid paraffin under a coverslip, and examined under the microscope.

- Cytology: tape strips were applied to the affected areas. The sticky side of the tape strip was applied to an area of skin several times. The tape was stained using Diff Quick, then the sticky side of the tape stuck on top of the slide, then examined under high power.

- Woods lamp: used to examine the dog for fluorescent hairs. This was performed in a dark room after allowing the lamp to warm up for ten minutes.

- Dermatophyte PCR: hairs were collected from the margin of the lesion with forceps. Hairs were sent in a sterile vial and in a plastic bag to Idexx Veterinary Laboratories.

For the second skin condition, skin scrape, cytology, trichogram, wood lamp and dermatophyte PCR were performed.

- Skin scrapes: taken from the feet, elbows, face. A no.10 scalpel blade moistened with liquid paraffin was used. The skin was squeezed, then the blade was used at a 30-degree angle to gently scrape the skin until capillary ooze appeared. The material obtained was spread on a slide in a drop of liquid paraffin, a coverslip applied and examined immediately.

- Trichogram: collected from the periocular area. See above for the procedure.

- $\quad$ Cytology: collected form face, paws, elbows and stifle. See above for the procedure. 
- Woods lamp: see above.

- $\quad$ Dermatophyte PCR: see above.

\section{Diagnostic Results and Interpretation}

\section{For the first skin condition:}

- Trichogram: the majority of hairs in the telogen phase, hair showed a normal structure. No evidence of narrowing of it or evidence of arthroconidia. No evidence of ectoparasites.

- Cytology: amorphous, glassy, transparent material and evidence of crystals, compatible with calcium salts (Figures 4, 5). Evidence of neutrophils, cocci and corneocytes. (Albanese, 2017)

- Woods lamp: negative.

- $\quad$ Dermatophyte PCR: negative.

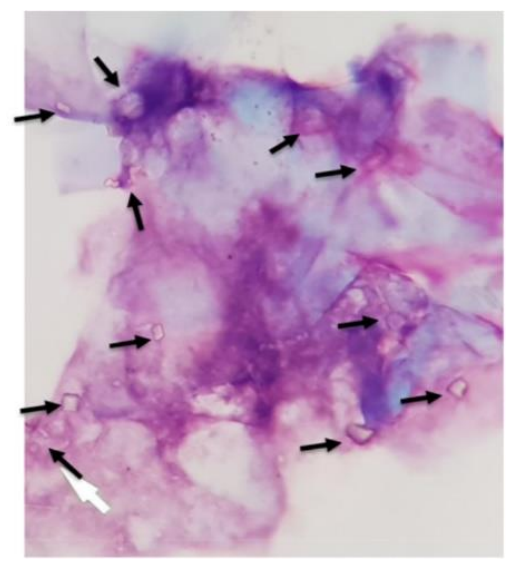

Figure 4: Cytology of the lesion where is possible to observe amorphous, glassy, transparent material and evidence of crystals (black arrows), compatible with calcium salts.

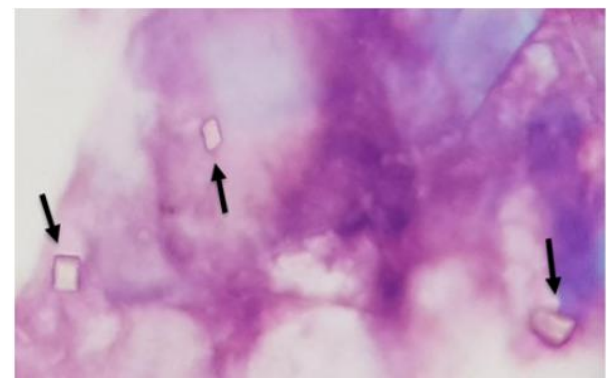

Figure 5: Particular of Figure 4, where are more clearly evident the crystals (black arrows).

For the second skin condition:

- $\quad$ Skin scrapes: evidence of numerous Demodex spp. mites from all the area sampled. No evidence of other ectoparasites. 
- Trichogram: no evidence of ectoparasites. Normal structure of the hair, no narrowing or presence of arthroconidia.

- Cytology: evidence of bacteria, cocci. Presence of neutrophils and corneocytes

- Woods lamp: negative.

- $\quad$ Dermatophyte PCR: negative.

\section{Final Diagnosis}

For the first skin condition, the final diagnosis was a suspect of CC considering the cytological findings and the clinical history of the dog.

Regarding the second skin condition, a diagnosis of demodicosis was made.

\section{Treatments}

The CC was managed reducing gradually the Prednidale ${ }^{\circledR}$ tablets from $0.5 \mathrm{mg} / \mathrm{Kg} /$ day to 0.2 $\mathrm{mg} / \mathrm{Kg} /$ day and, after $5 \mathrm{months}$, to $0.1 \mathrm{mg} / \mathrm{Kg} /$ day. Considering the presence of bacteria and the clinical aspect of the lesion, systemic therapy with cephalexin 600mg, (22 mg/Kg) q12h (Rilexine®, Virbac) was started for 28 days. At day 28, the lesions were improved with no evidence of papules or ulcerative nodules. A repeat cytological examination was performed which identified persistence calcium deposition and crystals but no evidence of cocci. The course of cephalexin was extended for a further seven days. The dog was checked on a monthly base and after two months from the initial diagnosis, the skin was less erythematous, and the calcification was reduced. Five months after diagnosis most of the hairs had growth back although there was a very small area of white calcium deposition.

For the second skin condition, a single administration of a fluralaner containing chewable tablet (Bravecto®, MSD) was used and, due to the cocci infection, systemic empirical treatment with Rilexine ${ }^{\circledR}$ 600mg, (22 mg/Kg) q12h was prescribed, for 28 days. Topical treatment was not started, even if indicated in this case, because the owner refused to bathe the dog. At 28 days after the diagnosis, the previously affected areas of skin were less erythematous, and hair had started to grow back. The owner reported that the dog was not pruritic. The course of cephalexin was extended for a further seven days. At 56 days after diagnosis, the lesions had regressed completely, and the dog was not pruritic (Figure 6). A skin scrape was performed and was negative. At day 84 another skin scrape was performed and was also negative. 


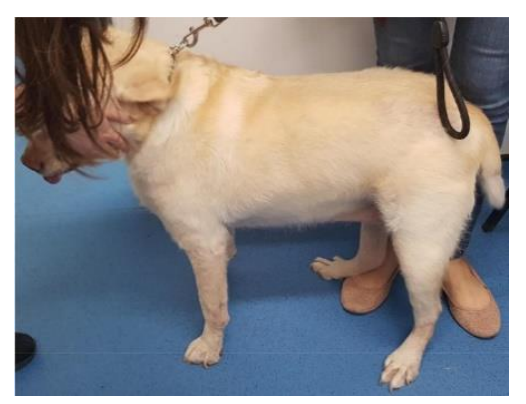

Figure 6: Dog 56 days after treatment for Demodex spp.

\section{Outcome}

Six months after the diagnosis of the presumptive CC and the reduction of steroids, the lesions were completely resolved, hair had grown back, and no other concerns were raised by the owner. For the demodicosis, after Braevecto® treatment, the clinical signs resolved. Her hypoadrenocorticism was well controlled when receiving $0.1 \mathrm{mg} / \mathrm{kg} /$ day of prednisolone. The prognosis for her skin conditions is good. Keeping the dog on a physiological dose of steroids should not cause a relapse of the CC. The demodicosis has been treated successfully and the owner decided to carry on with Braevecto® as routine flea and tick treatment.

\section{Discussion}

The present study discusses two different skin conditions presumably secondary to excessive exogenous administration of glucocorticoids. This dog was initially receiving a dose of $0.5 \mathrm{mg} / \mathrm{Kg} / \mathrm{day}$ prednisolone while a physiological dose is considered to be $0.1-0.25 \mathrm{mg} / \mathrm{kg} /$ day (Schaer, 2001; Peterson and Kintzer, 2009; Van Lanen and Sande, 2014; Lathan and Thompson, 2018). Studies report that iatrogenic hypercortisolism is the major cause for CC in dogs (Hsu et al., 1998; Doerr et al., 2013) and the role of cortisol in its development is considered a result of its gluconeogenic and protein catabolic activity (Doerr et al., 2013). The dose and duration of glucocorticoids associated with CC is unknown but, higher doses, administered for a long time, are thought to induce changes more rapidly (Hsu et al., 1998). According to Doerr et al., (2013), CC secondary to hypercortisolism can develop after seven weeks of corticosteroid therapy. Frazier et al., (1998) report that cutaneous lesions resulting from iatrogenic hypercortisolism, including $\mathrm{CC}$, usually regress within two to four months after discontinuing corticosteroid therapy. These reports are consistent with the findings of this care report, considering the lesions appeared six months after the beginning of prednisolone treatment and reduction of prednisolone to a physiological dose improved the clinical signs within 2 months, with complete resolution in about six months. The underline pathogenesis of CC, in this case, is speculative considering that, unfortunately, owing to financial constraints, further diagnostic test like a biopsy, was not performed, making the 
diagnosis based on clinical signs and cytology only.

Specific systemic conditions such as leishmaniosis, hypothyroidism, hyperadrenocorticism, neoplasia, immunosuppressive treatments or excessive exogenous glucocorticoids can potentiate Demodex mite populations (Mueller, 2004; Coatesworth, 2011; Ferrer et al., 2014) and predispose to a clinical manifestation of demodicosis. Considering not all dogs treated with corticosteroids or immunosuppressive drugs develop demodicosis, it is plausible to think that cofactors or specific genetic profiles are necessary for the development of clinical demodicosis (Ferrer et al., 2014). In this case, is likely chronic excessive exogenous glucocorticoids contributed to the development of demodicosis, but it is possible, that an independent condition contributed to the infestation. Is interesting to observe that the trichogram examination of the hair collected from the periocular area was negative. This finding is in line with the information reported in the article of Mueller (2004) and Arsenovic et al., (2015) where trichograms revealed Demodex mites in 50\% of dogs with demodicosis. Therefore, a negative trichogram does not exclude demodicosis, and a deep skin scrape or a biopsy remains the most sensitive method to obtain a diagnosis (Mueller, 2004; Arsenovic et al., 2015).

Regarding the treatment for demodicosis in UK, only three drugs are licensed: amitraz bathing (Aludex®, MSD); a spot-on formulation imidacloprid/moxidectin (Advocate®, Bayer; Prinovox®, Virbac) (Cerundolo, 2016); and, recently registered, sarolaner chewable tablet (Simparica ${ }^{\circledR}$, Zoetis). Alongside these medications are other off label drugs: spot-on formulation containing fipronil/amitraz/(S)methoprene (Certifect $\AA$, Merial); Bravecto®; afoxolaner chewable tablet (NexGard $®$, Merial); iver mectin

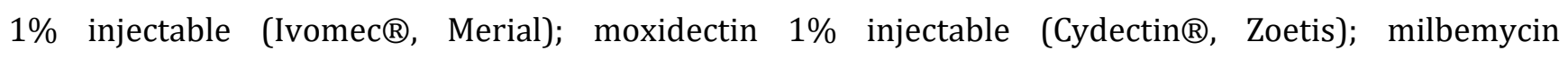
oxime/praziquantel (Milbemax®, Elanco; Milpro®, Virbac; Milquantel®, MSD) (Cerundolo, 2016). In this case report, Bravecto® was chosen. This because Simparica ${ }^{\circledR}$ was not yet registered for demodicosis treatment and Aludex® is associated with different side effects (Cerundolo, 2016) and is more laborious to use compared with other drugs. In a study of Fourie et al, (2015), was evaluated the efficacy of a single oral administration of Bravecto® chewable tablets to dogs with naturally acquired generalized demodicosis resulted in significantly lower mean mite counts at 56 and 84 days after treatment associated with a reduction in the extent and severity of the skin changes. These data are in line with that observed in this case report because, a single administration of Bravecto ${ }^{\circ}$ chewable tablet, caused a reduction on clinical signs and mite population at 56 and 84 days post-treatment.

Advocate ${ }^{\circledR}$ was no choice because the owner was reluctant to apply a pipette on a weekly base. The owner was informed that Bravecto® was off-license for Demodex spp. treatment and the consensus agreement was signed. 


\section{Acknowledgment}

I would like to sincerely thank you Dr Christopher Scudder for the English review and for his critical comment on the internal medicine part; and the Dr Filippo De Bellis for his corrections and critical comments on the dermatological part.

\section{Conclusion}

This case report shows an atypical situation where the inappropriate use of steroids predisposed to a multiple skins condition. Unfortunately, in the literature, there are no case reports of CC secondary to hypoadrenocorticism, and owing to owner financial constraints, additional investigations could not be performed. However, the response to prednisolone dose reduction supports the notion that iatrogenic hyperadrenocorticism was the cause.

\section{References}

Albanese F. Cytology of canine and feline non-neoplastic skin diseases. 1st ed. Switzerland: Springer International Publishing 2017; pp. 108-116.

Arsenovic M, Pezo L, Vasic N, CIric R, Stefanovic M. The main factors influencing canine demodicosis treatments outcome and determination of optimal therapy. Parasitology Research 2015; 114: 2415-2426.

Cereundolo R. Treatment of canine demodicosis. In Practice 2016; 38: 675-687.

Coatesworth J. Canine demodicosis consideration. Vet Times [online], 2011. Available at: https://www.vettimes.co.uk > uploads > wp-post-to-pdf-enhanced-cache [Accessed 17 November 2019].

Doerr AK, Outerbridge CA, White SD, Kass PH, Shiraki R, Lam AT, Affolter VK. Calcinosis cutis in dogs: histopathological and clinical analysis of 46 cases. Veterinary Dermatology 2013; 24: 355-361.

Ferrer L, Ravera I, Silbermayr K. Immunology and pathogenesis of canine demodicosis. Veterinary Dermatology 2014; 25: 427-435.

Fourie JJ, Liebenberg JE, Horak IG, Taenzler J, Heckeroth AR, Frénais R. Efficacy of orally administered fluralaner (BravectoTM) or topically applied imidacloprid/moxidectin (Advocateß) against generalized demodicosis in dogs. Parasites \& Vectors 2015; 8: 187.

Frazier KS, Hullinger GA, Liggett AD, Hines II ME. Multiple cutaneous metaplastic ossification associated with iatrogenic hyperglucocorticoidism. Journal of Veterinary Diagnostic Investigation 1998; 10: 303-307.

Holahan ML, Loft KE, Swenson CL, Martinez-Ruzaf L. Generalized calcinosis cutis associated with disseminated paecilomycosis in dog. Veterinary Dermatology 2006; 19: 368-372.

Hsu K, Snead E, Davies J, Carr A. Iatrogenic Hyperadrenocorticism, calcinosis cutis and myocardial infarction in a dog treated for IMT. Journal of the American Animal Hospital Association 2012; 48: 209-215.

Huang HP, Yang HLY, Liang SL, Lien YH, Chen KY. Iatrogenic Hyperadrenocorticism in 28 Dogs. Journal of the American Animal Hospital Association 1999; 35: 200-207. 
Jang HJ, Kang MH, Sur JH, Park HM. Minocycline as a treatment of dog with calcinosis cutis. Korean Journal of Veterinary Research 2013; 53: 253-256.

Koch SN. Updates on the management of canine demodicosis. Today's Veterinary Practice [online], 2017. Available at: https://todaysveterinarypractice.com/dermatology-detailsupdates-management-canine-demodicosis/ [Accessed 17 November 2019].

Lathan P and Thompson AL. Management of hypoadrenocorticism (Addison's disease) in dogs. Veterinary Medicine: Research and Reports 2018; 9: 1-10.

Mueller RS. Treatment protocols for demodicosis: an evidence-based review. Veterinary Dermatology 2004; 15 : 75-89.

Munday JS, Bergen DJ, Roe WD. Generalized calcinosis cutis associated with probable leptospirosis in a dog. Veterinary Dermatology 2005; 16: 401-406.

Peterson ME and Kintzer PP. Hypoadrenocorticism. In: J.D. Bonagura, D.C. Twedt, ed. Kirk's Current Veterinary Therapy. 14th ed. St. Louis: Elsevier. 2009; pp. 231-235.

Schaer M, Ginn PE, Fox LE, Leon J, Ramirez FM. Severe calcinosis cutis associated with treatment of hypoparathyroidism in a dog. Journal of the American Animal Hospital Association 2001; 37: 364-369.

Tan RM, Stern AW, White AG, Campbell KL, Novak K. Pathology in practice. Calcinosis cutis. Journal of the American Veterinary Medical Association 2013; 243: 347-349.

Van Lanen K and Sande A. Canine Hypoadrenocorticism: Pathogenesis, Diagnosis, and Treatment. Topics in Companion Animal Medicine 2014; 29: 88-95.

Volk AV, Volk HA, Rest JR, Loderstedt S, Bond R. Calcinosis cutis at cytarabine injection site in three dogs receiving prednisolone. The Veterinary Record 2015; 171: 327-329.

WSAVA.org. WSAVA Official Website [Online]. Body condition score, 2013. Available at: https://www.wsava.org/sites/default/files/Body\%20 condition\%20score $\% 20$ chart $\% 20$ dogs.pdf 